\title{
COMPLEX CYCLES ON REAL ALGEBRAIC MODELS OF A SMOOTH MANIFOLD
}

\author{
J. BOCHNAK AND W. KUCHARZ
}

(Communicated by Frederick R. Cohen)

\begin{abstract}
ABsTRACr. Let $M$ be a compact connected orientable $C^{\infty}$ submanifold of $\mathbb{R}^{n}$ with $2 \operatorname{dim} M+1 \leq n$. Let $G$ be a subgroup of $H^{2}(M, \mathbb{Z})$ such that the quotient group $H^{2}(M, \mathbb{Z})$ has no torsion. Then $M$ can be approximated in $\mathbb{R}^{n}$ by a nonsingular algebraic subset $X$ such that $H_{\mathrm{C}-\text { alg }}^{2}(X, \mathbb{Z})$ is isomorphic to $G$. Here $H_{\mathrm{C} \text {-alg }}^{2}(X, \mathbb{Z})$ denotes the subgroup of $H^{2}(X, \mathbb{Z})$ generated by the cohomology classes determined by the complex algebraic hypersurfaces in a complexification of $X$.
\end{abstract}

\section{INTRODUCTION}

In $[6,14]$ we have defined a contravariant functor $H_{\mathbb{C}-a l g}^{\text {even }}(\cdot, \mathbb{Z})$ from affine nonsingular real algebraic varieties to graded rings. If $X$ is an affine nonsingular real algebraic variety, then $H_{\mathbb{C}-a l g}^{\text {even }}(X, \mathbb{Z})=\bigoplus_{k \geq 0} H_{\mathbb{C} \text {-alg }}^{2 k}(X, \mathbb{Z})$ is a graded subring of the graded ring $H^{\text {even }}(X, \mathbb{Z})=\bigoplus_{k \geq 0} H^{2 k}(X, \mathbb{Z})$ generated, roughly speaking, by the cohomology classes determined by the complex algebraic cycles on a nonsingular complexification of $X$ (cf. [7] and $\S 1$ for details). The functor $H_{\mathbb{C} \text {-alg }}^{\text {even }}(\cdot, \mathbb{Z})$ has played a crucial role in the study of vector bundles over real algebraic varieties $[6,9,14]$ and real algebraic morphisms [11, 12]. Further applications will be discussed in our subsequent papers.

A well-known theorem of Tognoli (cf. [7, Theorem 14.1.10 or [20] or [25]) asserts that given a compact $C^{\infty}$ submanifold $M$ of $\mathbb{R}^{n}$ with $2 \operatorname{dim} M+1 \leq n$, one can find a $C^{\infty}$ embedding $e: M \rightarrow \mathbb{R}^{n}$, arbitrarily close in the $C^{\infty}$ topology to the inclusion mapping $M \hookrightarrow \mathbb{R}^{n}$ such that $X=e(M)$ is a nonsingular algebraic subvariety of $\mathbb{R}^{n}$ (cf. also recent result $[3,24]$ ). The variety $X$ is referred to as an algebraic subvariety of $\mathbb{R}^{n}$ approximating $M$. It is known that the class $\mathscr{A}(M)$ of algebraic subvarieties of $\mathbb{R}^{n}$ approximating $M$ contains infinitely many varieties that are mutually birationally nonisomorphic (cf. [10, Corollary 3.3]). The study of algebro-geometric properties of the varieties in $\mathscr{A}(M)$ is important for better understanding relations between differential topology and real algebraic geometry (cf. $[2,1,6,12,9])$. In this paper we investigate the behavior of the groups $H_{\mathbb{C} \text {-alg }}^{2 k}(X, \mathbb{Z})$ for $X$ in $\mathscr{A}(M)$. We prove

Received by the editors December 5, 1989.

1991 Mathematics Subject Classification. Primary 57R19, 14G30, 14 C99.

Research supported by NSF Grant DMS-8905538. 
that for some $X$ in $\mathscr{A}(M)$, one has $H_{\mathbb{C}-a l g}^{2 k}(X, \mathbb{Z}) \otimes_{\mathbb{Z}} \mathbb{Q}=H^{2 k}(X, \mathbb{Z}) \otimes_{\mathbb{Z}} \mathbb{Q}$ for all $k \geq 0$ (cf. Theorem 1.1). Also, given a subgroup $G$ of $H^{2}(M, \mathbb{Z})$ such that the factor group $H^{2}(M, \mathbb{Z}) / G$ is torsionfree, and assuming $M$ connected and orientable, we show the existence of $X$ in $\mathscr{A}(M)$ with $H_{\mathbb{C}-a l g}^{2}(X, \mathbb{Z})$ isomorphic to $G$ (cf. Theorem 1.2).

For the background material on real algebraic geometry the reader may consult [7]. Unless otherwise stated, algebraic subvarieties are assumed to be Zariski closed in the ambient variety.

\section{MAIN RESUlT}

We first recall a definition of the functor $H_{\mathbb{C} \text {-alg }}^{\text {even }}(\cdot, \mathbb{Z})$.

Let $X$ be an affine nonsingular real algebraic variety. Let $\varphi: X \rightarrow \mathbb{R} P^{n}$ be an algebraic embedding of $X$ in the real projective space $\mathbb{R} P^{n}$, that is, $\varphi(X)$ is a Zariski locally closed algebraic subvariety of $\mathbb{R} P^{n}$ and $\varphi$ induces a biregular isomorphism from $X$ onto $\varphi(X)$. Consider $\mathbb{R} P^{n}$ as a subset of the complex projective space $\mathbb{C} P^{n}$, and let $V$ be the Zariski (complex) closure of $\varphi(X)$ in $\mathbb{C} P^{n}$. Let $U$ be a Zariski (complex) neighborhood of $\varphi(X)$ in the set of nonsingular points of $V$. Given a complex algebraic subvariety $W$ of $U$, we let $[W]$ denote its fundamental class in the Borel-Moore homology group $H_{*}^{\mathrm{BM}}(W, \mathbb{Z})$ of $W$ (cf. [13] or [16, Chapter 19]). Let $a_{W}$ be the element of the cohomology group $H^{*}(U, \mathbb{Z})$ Poincare dual to the image of $[W]$ under the homomorphism $H_{*}^{\mathrm{BM}}(W, \mathbb{Z}) \rightarrow H_{*}^{\mathrm{BM}}(U, \mathbb{Z})$ induced by the inclusion mapping $W \hookrightarrow U$. Denote by $H_{\mathrm{alg}}^{2 k}(U, \mathbb{Z})$ the subgroup of $H^{2 k}(U, \mathbb{Z})$ generated by all elements of the form $a_{w}$, where $W$ runs through the set of complex algebraic subvarieties of $U$ of codimension $k$. It is known that $H_{\mathrm{alg}}^{\text {even }}(U, \mathbb{Z})=\bigoplus_{k \geq 0} H_{\mathrm{alg}}^{2 k}(U, \mathbb{Z})$ is a graded subring of $H^{\text {even }}\left(U, \mathbb{Z}\right.$ ) (cf. [13] or [16]). Define $\varphi_{U}: X \rightarrow U$ by $\varphi_{U}(x)=\varphi(x)$ for $x$ in $X$ and set

$$
\begin{aligned}
& H_{\mathbb{C} \text {-alg }}^{2 k}(X, \mathbb{Z})=\varphi_{U}^{*}\left(H_{\mathrm{alg}}^{2 k}(U, \mathbb{Z})\right), \\
& H_{\mathbb{C} \text {-alg }}^{\text {even }}(X, \mathbb{Z})=\bigoplus_{k \geq 0} H_{\mathbb{C} \text {-alg }}^{2 k}(X, \mathbb{Z}) .
\end{aligned}
$$

One shows that the graded ring $H_{\mathbb{C} \text {-alg }}^{\text {even }}(X, \mathbb{Z})$ does not depend on the choice of the embedding $\varphi$ and the neighborhood $U$ (cf. $[6, \S 3]$ ).

The group $H_{\mathbb{C}-a l g}^{2}(X, \mathbb{Z})$ has a simple, purely algebraic, interpretation. Namely, let $\mathscr{R}(X, \mathbb{C})$ denote the ring of regular functions from $X$ to $\mathbb{C}$ (cf. [7, Chapter 12]). Then $H_{\mathbb{C} \text {-alg }}^{2}(X, \mathbb{Z})$ is canonically isomorphic to the Picard group $\operatorname{Pic}(\mathscr{R}(X, \mathbb{C}))$ of $\mathscr{R}(X, \mathbb{C})$ (cf. [6, Remark 5.4]).

If $f: X \rightarrow Y$ is a regular mapping between affine nonsingular real algebraic varieties, then the induced homomorphism $f^{*}: H^{*}(Y, \mathbb{Z}) \rightarrow H^{*}(X, \mathbb{Z})$ maps $H_{\mathbb{C}-\text { alg }}^{\text {even }}(Y, \mathbb{Z})$ into $H_{\mathbb{C} \text {-alg }}^{\text {even }}(X, \mathbb{Z})($ cf. $[6, \S 3])$.

We identify $H^{*}(X, \mathbb{Z}) \otimes \mathbb{Q}$ with $H^{*}(X, \mathbb{Q})$ and consider $H_{\mathbb{C}-a l g}^{\text {even }}(X, \mathbb{Q})=$ $H_{\mathbb{C}-\text { alg }}^{\text {even }}(X, \mathbb{Z}) \otimes \mathbb{Q}$ as a subring of $H^{\text {even }}(X, \mathbb{Q})$.

Now we can state our results.

Theorem 1.1. Let $M$ be a compact $C^{\infty}$ submanifold of $\mathbb{R}^{n}$ with $2 \operatorname{dim} M+1 \leq$ $n$. Then there exists a $C^{\infty}$ embedding $e: M \rightarrow \mathbb{R}^{n}$, arbitrarily close in the $C^{\infty}$ topology to the inclusion mapping $M \hookrightarrow \mathbb{R}^{n}$, such that $X=e(M)$ is a 
nonsingular algebraic subvariety of $\mathbb{R}^{n}$ and the following conditions are satisfied:

(i) $H_{\mathbb{C}-a l g}^{\text {even }}(X, \mathbb{Q})=H^{\text {even }}(X, \mathbb{Q})$,

(ii) $H_{\mathbb{C} \text {-alg }}^{2}(X, \mathbb{Z})=H^{2}(X, \mathbb{Z})$.

This theorem can be easily derived from [6] and is recorded here for the sake of completeness, since our more interesting and more precise result below concerns only $H_{\mathbb{C}-a l g}^{2}$.

Theorem 1.2. Let $M$ be a compact connected orientable $C^{\infty}$ submanifold of $\mathbb{R}^{n}$ with $2 \operatorname{dim} M+1 \leq n$. Let $G$ be a subgroup of $H^{2}(M, \mathbb{Z})$ such that the quotient group $H^{2}(M, \mathbb{Z}) / G$ is torsionfree. Then there exists a $C^{\infty}$ embedding $e: M \rightarrow \mathbb{R}^{n}$, arbitrarily close in the $C^{\infty}$ topology to the inclusion mapping $M \hookrightarrow \mathbb{R}^{n}$, such that $X=e(M)$ is a nonsingular algebraic subvariety of $\mathbb{R}^{n}$ and $h^{*}(G)=H_{\mathbb{C}-a l g}(X, \mathbb{Z})$ where $h: X \rightarrow M$ is the $C^{\infty}$ diffeomorphism defined by $h(e(m))=m$ for $m$ in $M$.

As an application we immediately obtain the following

Corollary 1.3. Let $M$ be a compact connected orientable $C^{\infty}$ submanifold of $\mathbb{R}^{n}$ with $2 \operatorname{dim} M+1 \leq n$ and the cohomology group $H^{2}(M, \mathbb{Z})$ torsionfree. Then there exists a $C^{\infty}$ embedding $e: M \rightarrow \mathbb{R}^{n}$, arbitrarily close in the $C^{\infty}$ topology to the inclusion mapping $M \hookrightarrow \mathbb{R}^{n}$, such that $X=e(M)$ is a nonsingular algebraic subvariety of $\mathbb{R}^{n}$ and the ring $\mathscr{R}(X, \mathbb{C})$ is a unique factorization domain.

Proof. By Theorem 1.2 there exists a $C^{\infty}$ embedding $e: M \rightarrow \mathbb{R}^{n}$, arbitrarily close in the $C^{\infty}$ topology to the inclusion mapping $M \hookrightarrow \mathbb{R}^{n}$, such that $X=$ $e(M)$ is a nonsingular algebraic subvariety of $\mathbb{R}^{n}$ and $H_{\mathbb{C} \text {-alg }}^{2}(X, \mathbb{Z})=0$. Since $H_{\mathbb{C} \text {-alg }}^{2}(X, \mathbb{Z})$ is isomorphic to $\operatorname{Pic}(\mathscr{R}(X, \mathbb{C}))$, we obtain $\operatorname{Pic}(\mathscr{R}(X, \mathbb{C}))=0$. It follows that the ring $\mathscr{R}(X, \mathbb{C})$ is a unique factorization domain.

Remark 1.4. In Corollary 1.3 the assumption that $H^{2}(M, \mathbb{Z})$ is torsionfree cannot be dropped. Indeed, if $M$ is a compact, connected, nonorientable surface of odd genus, then $H_{\mathbb{C} \text {-alg }}^{2}(X, \mathbb{Z})=H^{2}(X, \mathbb{Z}) \cong \mathbb{Z} / 2$ for each affine nonsingular real algebraic variety $X$ homeomorphic to $M$ (cf. [8, Theorem 1.7(iii); 9, Proposition 1.2]).

\section{Proof OF THEOREM 1.1}

Let $X$ be an affine real algebraic variety. Let $\mathbb{F}=\mathbb{R}$ or $\mathbb{C}$. An algebraic $\mathbb{F}$-vector bundle over $X$ is said to be strongly algebraic if it is algebraically isomorphic to a subbundle of a product vector bundle $X \times \mathbb{F}^{n}$ for some $n$ (cf. [7, Chapter 12] for an exposition of the theory of strongly algebraic vector bundles). A topological $\mathbb{F}$-vector bundle over $X$ is said to admit an algebraic structure if it is topologically isomorphic to a strongly algebraic vector bundle over $X$ (cf. [6]). Denote by $K_{\mathrm{F}}(X)$ the Grothendieck group of topological $\mathbb{F}$-vector bundles over $X$ (cf. [19]) and by $K_{\mathbb{F} \text {-alg }}(X)$ the subgroup of $K_{\mathbb{F}}(X)$ generated by the classes of $\mathbb{F}$-vector bundles admitting an algebraic structure.

Proof of Theorem 1.1. By [4, Theorem 4.2] there exists a $C^{\infty}$ embedding $e: M \rightarrow \mathbb{R}^{n}$, arbitrarily close in the $C^{\infty}$ topology to the inclusion mapping $M \hookrightarrow \mathbb{R}^{n}$, such that $X=e(M)$ is a nonsingular algebraic subvariety of $\mathbb{R}^{n}$ and 
$K_{\mathbb{F} \text {-alg }}(X)=K_{\mathbb{F}}(X)$. (Strictly speaking, the above statement is proved in [4] for $\mathbb{F}=\mathbb{R}$. However, in order to obtian a proof for $\mathbb{F}=\mathbb{C}$ only a straightforward modification is required. Below we use this result with $\mathbb{F}=\mathbb{C}$.) It is known that the isomorphism

$$
\text { ch: } K_{\mathbb{C}}(X) \otimes \mathbb{Q} \rightarrow H^{\text {even }}(X, \mathbb{Q})
$$

induced by the Chern character (cf. [21]) maps $K_{\mathbb{C} \text {-alg }}(X) \otimes \mathbb{Q}$ onto $H_{\mathbb{C} \text {-alg }}^{\text {even }}(X, \mathbb{Q})$ (cf. [6, Proposition 5.9]). Hence (i) follows at once.

Since the homomorphism $c_{1}: K_{\mathbb{C}}(X) \rightarrow H^{2}(X, \mathbb{Z})$ determined by the first Chern class is surjective (cf. [19, Chapter 16, Theorem 3.4]) and maps $K_{\mathbb{C} \text {-alg }}(X)$ on to $H_{\mathbb{C} \text {-alg }}^{2}(X, \mathbb{Z})$ (cf. [6, Theorem 5.2 and Remark 5.4]), we also immediately obtain (ii).

\section{Proof of Theorem 1.2}

We first need a few auxiliary results.

Let, as before, $\mathbb{F}=\mathbb{R}$ or $\mathbb{C}$. Denote by $G_{n, p}(\mathbb{F})$ the Grassmann variety of $p$-dimensional vector subspaces of $\mathbb{F}^{n}$. Recall that $G_{n, p}(\mathbb{F})$ is an affine nonsingular real algebraic variety [7, Theorem 3.4.4, Proposition 3.4.8], and the universal vector bundle $\gamma_{n, p}(\mathbb{F})$ over $G_{n, p}(\mathbb{F})$ is strongly algebraic [7, Theorem 12.1.7, p. 280].

Let $X$ be an affine nonsingular real algebraic variety. An algebraic subvariety $Y$ of $X$ is said to be quasi-regular if for each point $x$ in $X$ the ideal of real analytic function-germs $(X, x) \rightarrow \mathbb{R}$ vanishing on $Y$ is generated by regular functions on $X$ vanishing on $Y$. One can show that a union of finitely many nonsingular algebraic subvarieties of $X$ is quasi-regular [23, p. 75].

Lemma 3.1. Let $X$ be a compact affine nonsingular real algebraic variety, and let $Y$ be a quasi-regular algebraic subvariety of $X$. Let $f: X \rightarrow G_{n, p}(\mathbb{F})$ be a $C^{\infty}$ mapping whose restriction $f \mid Y$ to $Y$ is a regular mapping. If the pullback vector bundle $f^{*} \gamma_{n, p}(\mathbb{F})$ admits an algebraic structure, then there exists a regular mapping $g: X \rightarrow G_{n, p}(\mathbb{F})$, arbitrarily close in the $C^{0}$ topology to $f$, such that $g|Y=f| Y$.

Proof. Let $\gamma=\gamma_{n, p}(\mathbb{F})$, and let $\xi$ be a strongly algebraic $\mathbb{F}$-vector bundle over $X$ which is topologically, hence also $C^{\infty}$, isomorphic to $f^{*} \gamma$. Take a $C^{\infty}$ section $u: X \rightarrow \operatorname{Hom}\left(\xi, f^{*} \gamma\right)$ of $\operatorname{Hom}\left(\xi, f^{*} \gamma\right)$ such that $u(x): \xi_{x} \rightarrow$ $\left(f^{*} \gamma\right)_{x}$ is an isomorphism of fibres for all $x$ in $X$. Since $f \mid Y$ is a regular mapping, it follows that $\operatorname{Hom}\left(\xi, f^{*} \gamma\right) \mid Y$ is a strongly algebraic $\mathbb{F}$-vector bundle over $Y$ (cf. [7, Proposition 12.1.8]). By [7, Theorem 12.3.1] $u \mid Y$ can be approximated in the $C^{0}$ topology by regular sections $v: Y \rightarrow \operatorname{Hom}\left(\xi, f^{*} \gamma\right) \mid Y$. If $v$ is sufficiently close to $u \mid Y$, then it extends to a $C^{\infty}$ section, say $w$, of $\operatorname{Hom}\left(\xi, f^{*} \gamma\right)$ such that $w$ is close in the $C^{0}$ topology to $u$. In particular we may assume that $w(x): \xi_{x} \rightarrow\left(f^{*} \gamma\right)_{x}$ is an isomorphism for all $x$ in $X$.

Consider $f^{*} \gamma$ as a $C^{\infty} \mathbb{F}$-vector subbundle of the product vector bundle $\varepsilon=X \times \mathbb{F}^{n}$, and let $i: f^{*} \gamma \rightarrow \varepsilon$ be the inclusion homomorphism. By [24] there exists a regular section $s: X \rightarrow \operatorname{Hom}(\xi, \varepsilon)$, arbitrarily close in the $C^{\infty}$ topology to $i \circ w$, such that $s|Y=i \circ w| Y$ (in particular, we may assume that $s(x): \xi_{x} \rightarrow \varepsilon_{x}$ is a monomorphism for all $x$ in $\left.X\right)$. Define $g: X \rightarrow G_{n, p}(\mathbb{F})$ by $g(x)=\rho\left(s(x)\left(\xi_{x}\right)\right)$ for $x$ in $X$, where $\rho: X \times \mathbb{F}^{n} \rightarrow \mathbb{F}^{n}$ is the standard 
projection. By construction, $g$ is a regular mapping (cf. [7, Proposition 3.4.9]) close in the $C^{0}$ topology to $f$ and $g|Y=f| Y$.

Lemma 3.2. Let $M$ be a compact $C^{\infty}$ submanifold of $\mathbb{R}^{q}$. Let $Y_{1}, \ldots, Y_{k}$ be compact $C^{\infty}$ submanifolds of $M$ that are in general position (considered as submanifolds of $M)$. Let $f: M \rightarrow G_{n, p}(\mathbb{F})$ be a $C^{\infty}$ mapping. Assume that $Y_{i}$ is a nonsingular algebraic subvariety of $\mathbb{R}^{q}$ and the pullback vector bundle $\left(f \mid Y_{i}\right)^{*} \gamma_{n, p}(\mathbb{F})$ over $Y_{i}, i=1, \ldots, k$, admits an algebraic structure. Then there exists a $C^{\infty}$ mapping $g: M \rightarrow G_{n, p}(\mathbb{F})$, arbitrarily close in the $C^{0}$ topology to $f$, such that $g \mid Y$ is a regular mapping where $Y=Y_{1} \cup \cdots \cup Y_{k}$.

Proof. Set $Y^{0}=\varnothing$ and $Y^{l}=Y_{1} \cup \cdots \cup Y_{l}$ for $l=1, \ldots, k$. Let us fix $l$, $0 \leq l \leq k-1$, and suppose that one can find a $C^{\infty}$ mapping $g^{l}: M \rightarrow G_{n, p}$, arbitrarily close in the $C^{0}$ topology to $f$, such that $g^{l} \mid Y^{l}$ is a regular mapping. We claim that there exists a $C^{\infty}$ mapping $g^{l+1}: M \rightarrow G_{n, p}(\mathbb{F})$, arbitrarily close in the $C^{0}$ topology to $f$, such that $g^{l+1} \mid Y^{l+1}$ is a regular mapping.

Indeed, observe that $Y_{1} \cap Y_{l+1}, \ldots, Y_{l} \cap Y_{l+1}$ are nonsingular subvarieties of $Y_{l+1}$ that are in general position (considered as $C^{\infty}$ submanifolds to $Y_{l+1}$ ). In particular, by Lemma 3.1, there exists a regular mapping $\varphi: Y_{l+1} \rightarrow G_{n, p}(\mathbb{F})$, arbitrarily close in the $C^{0}$ topology to $g^{l} \mid Y_{l+1}$, such that $\varphi\left|Z=g^{l}\right| Z$ where $Z=\bigcup_{i=1}^{l}\left(Y_{i} \cap Y_{l+1}\right)$. Let $h: Y^{l+1} \rightarrow G_{n, p}(\mathbb{F})$ be a mapping defined by $h \mid Y^{l}=$ $g^{l}\left|Y^{l}, h\right| Y_{l+1}=\varphi$. Since, obviously, $h$ is continuous, it follows from [4, Lemma 3], that $h$ is regular. It suffices to define $g^{l+1}$ to be a $C^{\infty}$ extension to $M$ of $h$ that is close in the $C^{0}$ topology to $g^{l}$. Thus the claim is proved.

Now Lemma 3.2 follows from the claim by induction.

Lemma 3.3. Let $S$ be a compact orientable $C^{\infty}$ surface in $\mathbb{R}^{n}$. Assume that $S$ is connected if $n=5$. Then there exists a $C^{\infty}$ embedding $f: S \rightarrow \mathbb{R}^{n}$, arbitrarily close in the $C^{\infty}$ topology to the inclusion mapping $S \hookrightarrow \mathbb{R}^{n}$, such that $Y=f(S)$ is a nonsingular algebraic subvariety of $\mathbb{R}^{n}$ with $H_{\mathbb{C} \text {-alg }}^{2}(Y, \mathbb{Z})=0$.

Proof. Let $c=\operatorname{codim} S$. We claim that there exist compact $C^{\infty}$ hypersurfaces $H_{1}, \ldots, H_{c}$ of $\mathbb{R}^{n}$ that are in general position at each point of $S$ and satisfy $S=H_{1} \cap \cdots \cap H_{c}$.

If $c=1$, the claim is obvious. Let us assume then that $c \geq 2$, that is, $n \geq 4$. Identify $\mathbb{R}^{n}$, via the stereographic projection, with $S^{n} \backslash\{a\}$, where $S^{n}$ is the $n$-dimensional unit sphere and $a=(0, \ldots, 0,1)$. Given a positive integer $m$ with $m<n$, we also identify $S^{m}$ with $\left\{\left(x_{0}, \ldots, x_{n}\right) \in S^{n} \mid x_{m+1}=\cdots=x_{n}=\right.$ $0\}$. Observe that one can find a $C^{\infty}$ diffeomorphism $h: S^{n} \rightarrow S^{n}$ such that $h(S)$ is contained in $S^{4} \subset S^{n}$. (This is a standard result for $n \geq 6$ [18] and it follows from [13, p. 43] for $n=5$; to apply [13], we use the connectedness of $S$.) Replacing $S$ by $h(S)$, we may assume that $S$ is contained in $S^{4}$. This reduces the proof of the claim to the case $c=2$. One easily sees that the normal vector bundle of $S$ in $S^{4}$ is trivial. It follows that there exist a $C^{\infty}$ mapping $g: S^{4} \rightarrow S^{2}$ and a regular value $b$ of $g$ such that $S=g^{-1}(b)$. Let $\eta$ be an orientable $C^{\infty}$ real vector bundle over $S^{2}$, and let $u$ be a $C^{\infty}$ section of $\eta$ such that $\operatorname{rank} \eta=2, u$ is transverse to the zero section of $\eta$, and the set of zeros $u^{-1}(0)$ of $u$ is equal to $\{b\}$. Then the pullback section $g^{*} u$ of the pullback vector bundle $g^{*} \eta$ is transverse to the zero section of $g^{*} \eta$ and $\left(g^{*} u\right)^{-1}(0)=S$. Since $H^{2}\left(S^{4}, \mathbb{Z}\right)=0$, the vector bundle $g^{*} \eta$ is trivial (cf. $[19$, Chapter 16, Theorem 3.4]), and hence the claim follows. 
Now the conclusion of Lemma 3.3 follows at once from the claim and [6, Theorem 7.1, Remark 5.4].

We still need a few preliminary remarks.

If $\mathbb{C} P^{q}$ is considered as a real algebraic variety, then $H_{\mathbb{C} \text {-alg }}^{2}\left(\mathbb{C} P^{q}, \mathbb{Z}\right)=$ $H^{2}\left(\mathbb{C} P^{q}, \mathbb{Z}\right)$. Indeed, the universal complex bundle $\gamma_{q}$ over $\mathbb{C} P^{q}$ is a strongly algebraic complex line bundle and hence its first Chern class $c_{1}\left(\gamma_{q}\right)$ is in $H_{\mathbb{C} \text {-alg }}^{2}\left(\mathbb{C} P^{q}, \mathbb{Z}\right)$ (cf. [6, Theorem 5.3]). Now our claim is obvious since $c_{1}\left(\gamma_{q}\right)$ generates $H^{2}\left(\mathbb{C} P^{q}, \mathbb{Z}\right)$. It easily follows that $H_{\mathbb{C} \text {-alg }}^{2}(P, \mathbb{Z})=H^{2}(P, \mathbb{Z})$ where $P=\mathbb{C} P^{q(1)} \times \cdots \times \mathbb{C} P^{q(k)}$. Furthermore, every homology class in $H_{l}(P, \mathbb{Z} / 2)$, $l \geq 0$, can be represented by a nonsingular real algebraic subvariety of $P$. This last fact implies that every unoriented bordism class of $P$ is represented by a regular mapping $f: X \rightarrow P$ where $X$ is a compact affine nonsingular real algebraic variety $[2,20]$.

Proof of Theorem 1.2. Let $m=\operatorname{dim} M$. If $m \leq 1$, then $H^{2}(M, \mathbb{Z})=0$ and Theorem 1.2 is just a very special case of Tognoli's theorem (cf. Introduction), while for $m=2$ it follows from Lemma 3.3. Thus we assume below that $m \geq 3$.

Since the group $H^{2}(M, \mathbb{Z}) / G$ is torsionfree, there exists a torsionfree subgroup $H$ of $H^{2}(M, \mathbb{Z})$ such that $H^{2}(M, \mathbb{Z})=G \oplus H$. The bilinear mapping

$$
H^{2}(M, \mathbb{Q}) \times H^{m-2}(M, \mathbb{Q}) \rightarrow H^{m}(M, \mathbb{Q}), \quad(u, v) \rightarrow u \cup v,
$$

where $U$ stands for the cup product, is a dual pairing (cf. [15, Proposition 8.13]), and hence one can find elements $v_{1}, \ldots, v_{k}$ in $H^{m-2}(M, \mathbb{Z})$ with the property that for a given element $a$ in $H^{2}(M, \mathbb{Z})$ there is $a \cup v_{i}=0$ for $i=1, \ldots, k$ if and only if $a$ is in $G$. Let $\alpha_{i}$ be the homology class in $H_{2}(M, \mathbb{Z})$ Poincaré dual to $v_{i}$. By Thom's theorem [22, Theorem II.26], $\alpha_{i}$ can be represented by a compact oriented $C^{\infty}$ surface $S_{i}$ in $M$. Clearly, we may assume that the surfaces $S_{i}$ are in general position.

Note that if $r_{i}: H^{2}(M, \mathbb{Z}) \rightarrow H^{2}\left(S_{i}, \mathbb{Z}\right)$ is the homomorphism induced by the inclusion mapping $S_{i} \hookrightarrow M$, then given an element $w$ in $H^{2}(M, \mathbb{Z})$, we have

$$
r_{i}(w)=0 \text { for } i=1, \ldots, k \text { if and only if } w \in G .
$$

Moreover, by Lemma 3.3, there exists a $C^{\infty}$ embedding $f_{i}: S_{i} \rightarrow \mathbb{R}^{n}$, arbitrarily close in the $C^{\infty}$ topology to the inclusion mapping $S_{i} \hookrightarrow \mathbb{R}^{n}$, such that $f_{i}\left(S_{i}\right)$ is a nonsingular algebraic subvariety of $\mathbb{R}^{n}$ and $H_{\mathbb{C} \text {-alg }}^{2}\left(f_{i}\left(S_{i}\right), \mathbb{Z}\right)=0$. Now one can find a $C^{\infty}$ embedding $F: M \rightarrow \mathbb{R}^{n}$, close in the $C^{\infty}$ topology to the inclusion mapping $M \hookrightarrow \mathbb{R}^{n}$, such that $F \mid S_{i}=f_{i}$ for $i=1, \ldots, k$ (cf. [2, Lemma 2.9]). Hence replacing possibly $M$ by $F(M)$, we may assume that $S_{i}$ is a nonsingular algebraic subvariety of $\mathbb{R}^{n}$ and

$$
H_{\mathbb{C} \text {-alg }}^{2}\left(S_{i}, \mathbb{Z}\right)=0 \text { for } i=1, \ldots, k .
$$

Let $a_{1}, \ldots, a_{l}$ be generators of $G$. For each $j=1, \ldots, l$, one can find a positive integer $q(j)$ and a $C^{\infty}$ mapping $\varphi_{j}: M \rightarrow \mathbb{C} P^{q(j)}$ such that $\varphi_{j}^{*}\left(z_{j}\right)=$ $a_{j}$ where $z_{j}$ is a generator of $H^{2}\left(\mathbb{C} P^{q(j)}, \mathbb{Z}\right)$. Setting $P=\mathbb{C} P^{q(1)} \times \cdots \times \mathbb{C} P^{q(l)}$ and $\varphi=\left(\varphi_{1}, \ldots, \varphi_{l}\right)$, we get

$$
\varphi^{*}\left(H^{2}(P, \mathbb{Z})\right)=G
$$


Note that if $\gamma^{j}$ is the universal complex line bundle over $\mathbb{C} P^{q(j)}$, then in virtue of (1) and (3), we have $c_{1}\left(\left(\varphi_{j} \mid S_{i}\right)^{*} \gamma^{j}\right)=0$ in $H^{2}\left(S_{i}, \mathbb{Z}\right)$, and hence the complex line bundle $\left(\varphi_{j} \mid S_{i}\right)^{*} \gamma^{j}$ over $S_{i}$ is topologically trivial (cf. [19, Chapter 16, Theorem 3.4]). This implies (cf. Lemma 3.2) that there exists a $C^{\infty}$ mapping $\sigma: M \rightarrow P$, arbitrarily close in the $C^{0}$ topology to $\varphi$, such that $\sigma \mid S$ is a regular mapping where $S=S_{1} \cup \cdots \cup S_{k}$ (in particular, we may assume that $\sigma$ is homotopic to $\varphi$ ). Hence replacing possibly $\varphi$ by $\sigma$, we may assume that $\varphi \mid S$ is a regular mapping.

Let $g: M \rightarrow G_{n, n-m}(\mathbb{R})$ be the $C^{\infty}$ mapping defined by

$$
g(x)=\text { the orthogonal complement of } T_{x} M \text { in } \mathbb{R}^{n}
$$

for $x$ in $M$, where $T_{x} M$ is the tangent space to $M$ at $x$. Note that $\nu_{i}=$ $\left(g^{*} \gamma_{n, n-m}(\mathbb{R})\right) \mid S_{i}$ is the restriction to $S_{i}$ of the normal vector bundle of $M$ in $\mathbb{R}^{n}$. Since $M$ is orientable, the vector bundle $\nu_{i}$ is also orientable and hence admits an algebraic structure (cf. [7, Corollary 12.5.4]). By Lemma $3.2 \mathrm{~g} \mid S$ can be approximated in the $C^{0}$ topology by regular mappings.

Now the properties of $\varphi, S_{i}$, and $P$ established above and the fact that each unoriented bordism class of $P$ is represented by a regular mapping (cf. the paragraph preceding the proof of Theorem 1.2), allow us to apply the approximation theorem [4, Theorem 3] (cf. also [2, Propositon 2.8; 23]). It follows that one can find a $C^{\infty}$ embedding $e: M \rightarrow \mathbb{R}^{n}$, arbitrarily close in the $C^{\infty}$ topology to the inclusion mapping $M \hookrightarrow \mathbb{R}^{n}$, such that $X=e(M)$ is a nonsingular algebraic subvariety of $\mathbb{R}^{n}, e(x)=x$ for $x$ in $S$, and there exists a regular mapping $\psi: X \rightarrow P$ with $\psi \circ e$ close in the $C^{\infty}$ topology to $\varphi$ (in particular, we may assume that $\psi \circ e$ is homotopic to $\varphi$ ). Let $h: X \rightarrow M$ be the $C^{\infty}$ diffeomorphism defined by $h(e(m))=m$ for $m$ in $M$. By construction $h^{*}(G)=\psi^{*}\left(H^{2}(P, \mathbb{Z})\right)$. Since $\psi$ is a regular mapping and $H_{\mathbb{C} \text {-alg }}^{2}(P, \mathbb{Z})=H^{2}(P, \mathbb{Z})$, we obtain that $h^{*}(G)$ is contained in $H_{\mathbb{C} \text {-alg }}^{2}(X, \mathbb{Z})$. Moreover, if $\rho_{i}: H^{2}(X, \mathbb{Z}) \rightarrow H^{2}\left(S_{i}, \mathbb{Z}\right)$ is the homomorphism induced by the inclusion mapping, then given an element $w$ in $H^{2}(X, \mathbb{Z})$ we have

$$
\rho_{i}(w)=0 \text { for } i=1, \ldots, k \text { if and only if } w \in h^{*}(G)
$$

(cf. (1)). If $w$ is in $H_{\mathbb{C} \text {-alg }}^{2}(X, \mathbb{Z})$, then by $(2), \rho_{i}(w)=0$ for $i=1, \ldots, k$, and hence $w$ belongs to $h^{*}(G)$.

Thus, finally, $h^{*}(G)=H_{\mathbb{C} \text {-alg }}^{2}(X, \mathbb{Z})$, which finishes the proof.

\section{REFERENCES}

1. S. Akbulut and H. King, A relative Nash theorem, Trans. Amer. Math. Soc. 267 (1981), 465-481.

2. The topology of real algebraic sets with isolated singularities, Ann. of Math. (2) 113 (1981), 425-426.

3. __ On approximating submanifolds by algebraic sets, preprint, 1989.

4. R. Benedetti and A. Tognoli, Approximation theorems in real algebraic geometry, Algebra é Geometria, Boll. Un. Mat. Ital. Suppl. 2 (1980), 209-228.

5. __ On real algebraic vector bundles, Bull. Sci. Math. (2) 104 (1980), 89-1 12.

6. J. Bochnak, M. Buchner, and W. Kucharz, Vector bundles over real algebraic varieties, $K$ Theory J. 3 (1990), 271-298.

7. J. Bochnak, M. Coste, and M.-F. Roy, Géométrie algébrique réelle, Ergeb. Math. Grenzgeb. (3), vol. 12, Springer-Verlag, New York and Berlin, 1987. 
8. J. Bochnak and W. Kucharz, Algebraic approximation of mappings into spheres, Michigan Math. J. 34 (1987), 119-125.

9. $\longrightarrow$, Algebraic cycles and approximation theorems in real algebraic grometry, preprint, 1989.

10. __ Algebraic models of smooth manifolds, Invent. Math. 97 (1989), 585-611.

11. __, On real algebraic morphisms into even-dimensional spheres, Ann. of Math. (2) 128 (1988), 415-433.

12. _ On vector bundles and real algebraic morphisms, Real Analytic and Algebraic Geometry, Trento 1988, Lecture Notes in Math., vol. 1420, Springer-Verlag, Berlin and New York, 1990 , pp. 65-71.

13. A Borel and A. Haefliger, La classe d'homologie fondamentale d'un espace analytique, Bull. Soc. Math. France 89 (1961), 461-513.

14. M. Buchner and W. Kucharz, Algebraic vector bundles over real algebraic varieties, Bull. Amer. Math. Soc. 17 (1987), 279-282.

15. A. Dold, Lectures on algebraic topology, Grundlehren Math. Wiss., vol. 200, Springer, Berlin and New York 1980.

16. W. Fulton, Intersection theory, Ergeb. Math. Grenzgeb. (3), vol. 2, Springer-Verlag, New York and Berlin, 1984.

17. A. Haefliger, Plongement différentiables de variétés dans variétés, Comment. Math. Helv. 36 (1961), 47-82.

18. M. Hirsch, Differential topology, Graduate Texts in Math., vol. 33, Springer, Berlin and New York 1976.

19. M. Husemoller, Fibre bundles, 2nd ed., Springer, Berlin and New York 1975.

20. N. Ivanov, Approximation of smooth manifolds by real algebraic sets, Russian Math. Surveys 37 (1982), 1-59.

21. M. Karoubi, K-Theory, an introduction, Springer, Berlin and New York 1978.

22. R. Thom, Quelques propriétés globales des variétés differentiables, Comment. Math. Helv. 28 (1954), 17-86.

23. A. Tognoli, Algebraic approximation of manifolds and spaces, Séminaire Bourbaki 32e année, 1979/1980, no. 548, Lecture Notes in Math., vol. 842, Springer, Berlin, Heidelberg and New York, 1981, pp. 73-94.

24. __, Any compact differential submanifold of $\mathbb{R}^{n}$ has an algebraic approximation in $\mathbb{R}^{n}$, Topology 27 (1988), 205-210.

25. $\_$, Su una congettura di Nash, Ann. Scuola Norm. Sup. Pisa Cl. Sci. (4) 27 (1973), 167-185.

Department of Mathematics, VRije Universiteit, P.O. Box 7161, 1007 MC AMSTerdam, THE NETHERLANDS

Department of Mathematics, University of New Mexico, Albuquerque, N.M. 87131 